\title{
Pharmacological and dietary-supplement treatments for autism spectrum disorder: a systematic review and network meta-analysis
}

\author{
Spyridon Siafis ${ }^{1 *}$ (DD, Oğulcan Çıray ${ }^{2}$, Hui Wu' ${ }^{1}$, Johannes Schneider-Thoma' ${ }^{1}$, Irene Bighelli', Marc Krause ${ }^{3}$, \\ Alessandro Rodolico ${ }^{4}$, Anna Ceraso ${ }^{5}$, Giacomo Deste ${ }^{5}$, Maximilian Huhn ${ }^{1,6}$, David Fraguas ${ }^{7}$, \\ Antonia San José Cáceres ${ }^{14,15}$, Dimitris Mavridis ${ }^{9,10}$, Tony Charman ${ }^{11}$, Declan G. Murphy ${ }^{12}$, \\ Mara Parellada $8,13,14,15$, Celso Arango $8,13,14,15$ and Stefan Leucht ${ }^{1}$
}

\begin{abstract}
Background: There is still no approved medication for the core symptoms of autism spectrum disorder (ASD). This network meta-analysis investigated pharmacological and dietary-supplement treatments for ASD.

Methods: We searched for randomized-controlled-trials (RCTs) with a minimum duration of seven days in ClinicalTrials.gov, EMBASE, MEDLINE, PsycINFO, WHO-ICTRP (from inception up to July 8, 2018), CENTRAL and PubMed (up to November 3, 2021). The co-primary outcomes were core symptoms (social-communication difficulties-SCD, repetitive behaviors-RB, overall core symptoms-OCS) measured by validated scales and standardized-mean-differences (SMDs). Associated symptoms, e.g., irritability/aggression and attention-deficit/hyperactivity disorder (ADHD) symptoms, dropouts and important side-effects, were investigated as secondary outcomes. Studies in children/adolescents and adults were analyzed separately in random-effects pairwise and network meta-analyses.
\end{abstract}

Results: We analyzed data for 41 drugs and 17 dietary-supplements, from 125 RCTs ( $n=7450$ participants) in children/adolescents and 18 RCTs $(n=1104)$ in adults. The following medications could improve at least one core symptom domain in comparison with placebo: aripiprazole ( $k=6$ studies in analysis, SCD: SMD $=0.2795 \% \mathrm{Cl}[0.09,0.44]$, RB: $0.48[0.26,0.70])$, atomoxetine ( $k=3, \mathrm{RB}: 0.49[0.18,0.80])$, bumetanide ( $k=4, \mathrm{RB}: 0.35$ [0.09, 0.62], OCS: 0.61 [0.31, $0.91])$, and risperidone ( $k=4$, SCM: $0.31[0.06,0.55], \mathrm{RB}: 0.60[0.29,0.90] ; k=3$, OCS: $1.18[0.75,1.61])$ in children/adolescents; fluoxetine ( $k=1, \mathrm{RB}: 1.20[0.45,1.96])$, fluvoxamine $(k=1, \mathrm{RB}: 1.04[0.27,1.81])$, oxytocin $(k=6, \mathrm{RB}: 0.41[0.16$, $0.66])$ and risperidone $(k=1$, RB: $0.97[0.21,1.74])$ in adults. There were some indications of improvement by carnosine, haloperidol, folinic acid, guanfacine, omega-3-fatty-acids, probiotics, sulforaphane, tideglusib and valproate, yet imprecise and not robust. Confidence in these estimates was very low or low, except moderate for oxytocin. Medications differed substantially in improving associated symptoms, and in their side-effect profiles.

Limitations: Most of the studies were inadequately powered (sample sizes of 20-80 participants), with short duration (8-13 weeks), and about a third focused on associated symptoms. Networks were mainly star-shaped, and there were indications of reporting bias. There was no optimal rating scale measuring change in core symptoms.

\footnotetext{
*Correspondence: spyridon.siafis@tum.de

1 Department of Psychiatry and Psychotherapy, School of Medicine,

Technical University of Munich, Ismaningerstr. 22, 81675 Munich, Germany

Full list of author information is available at the end of the article
}

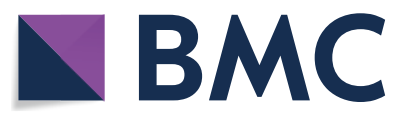

(c) The Author(s) 2022. Open Access This article is licensed under a Creative Commons Attribution 4.0 International License, which permits use, sharing, adaptation, distribution and reproduction in any medium or format, as long as you give appropriate credit to the original author(s) and the source, provide a link to the Creative Commons licence, and indicate if changes were made. The images or other third party material in this article are included in the article's Creative Commons licence, unless indicated otherwise in a credit line to the material. If material is not included in the article's Creative Commons licence and your intended use is not permitted by statutory regulation or exceeds the permitted use, you will need to obtain permission directly from the copyright holder. To view a copy of this licence, visit http://creativecommons.org/licenses/by/4.0/. The Creative Commons Public Domain Dedication waiver (http://creativeco mmons.org/publicdomain/zero/1.0/) applies to the data made available in this article, unless otherwise stated in a credit line to the data. 
Conclusions: Some medications could improve core symptoms, although this could be likely secondary to the improvement of associated symptoms. Evidence on their efficacy and safety is preliminary; therefore, routine prescription of medications for the core symptoms cannot be recommended.

Trial registration PROSPERO-ID CRD42019125317.

Keywords: Autism, Meta-analysis, Treatment, Response, Social communication, Restricted and repetitive behaviors, Irritability, ADHD, Anxiety, Caregiver stress

\section{Background}

Autism spectrum disorder (ASD) consists of heterogeneous conditions, which are characterized by social-communication difficulties, restricted interests/ repetitive behaviors and sensory abnormalities [1]. Behavioral interventions are the mainstay treatment [2]. Medications with different mechanisms of action have been examined in randomized controlled trials (RCTs) [3-5], and some of them have been found efficacious for associated symptoms, such as aripiprazole, risperidone and haloperidol for irritability, methylphenidate, atomoxetine, clonidine and guanfacine for attention-deficit/ hyperactivity disorder (ADHD) symptoms and melatonin for sleep disorders [2, 6]. However, prior late-stage clinical trials failed to identify efficacious treatments for the core symptoms of neurodevelopmental disorders $[5,7]$. Despite lack of clear evidence in efficacy, about half of the individuals with ASD receive psychotropic drugs [8]. The current synthesis of literature is restricted to medication classes or target symptoms [9-19], hence failing to combine the huge amount of recently conducted RCTs [3-5]. In order to better inform clinical practice and identify medications potentially efficacious for ASD, we combined evidence from pharmacological and dietarysupplement ASD trials in a network meta-analysis.

\section{Methods}

\section{Search strategy and selection criteria}

This network meta-analysis analyzed placebo-controlled and head-to-head RCTs on pharmacological/dietarysupplement interventions for ASD according to the PRISMA-NMA (Additional file 1: eAppendix-1) [20], and with PROSPERO-ID: CRD42019125317 (Additional file 1: eAppendix-2).

We searched ClinicalTrials.gov, EMBASE, MEDLINE, PsycINFO, WHO-ICTRP (from inception to July 8, 2018), CENTRAL and PubMed (last update on November 3, 2021), without restrictions in terms of language, document type, date/time and publication status (Additional file 1: eAppendix-3). Reference lists of included studies and reviews $[2,9-17,19,21-24]$ were inspected.

Participants should have a diagnosis of ASD according to standardized diagnostic criteria (e.g., DSM-III or newer versions) and/or validated diagnostic tools (e.g.,
ADI-R) [2], without restrictions in terms of age, sex, baseline severity and presence of genetic syndromes or other associated conditions (e.g., irritability, ADHD symptoms).

Any drug, dietary-supplement or placebo was eligible. We excluded augmentation and multimodal interventions (e.g., medications combined with risperidone or behavioral interventions) as well as other types of interventions (e.g., behavioral, elimination diets). The minimum duration of treatment was seven days, and there was no restriction in terms of dosing-schedule and route of administration. Multiple doses of the same intervention were combined [25] (Additional file 1: eAppendix-2.2).

Blinded and open RCTs were eligible. RCTs with a low or unclear risk of bias in random sequence generation and allocation concealment were eligible, yet we excluded trials with a high risk of bias in these domains [26]. Trials stated to be randomized but did not report the exact randomization methods (unclear risk of bias) were eligible, since poor reporting does not necessarily reflect the actual conducted methods [27-30] (Additional file 1: eAppendix-2.2). However, such trials were excluded in a post hoc sensitivity analysis. We included data only from the first phase of crossover studies in order to avoid carry-over effects [31], and we excluded discontinuation studies, studies published before 1980, or with a randomized sample smaller than ten participants [32].

At least two independent reviewers/contributors selected relevant records (SS, OC, HW, IB, MK, YZ, AC, $\mathrm{GD}$ and TF), extracted data from eligible studies into an Access database as well as evaluated risk of bias using the Cochrane risk-of-bias tool (SS, OC, AR, HW) [26]. Studies were rated as having a low, moderate or high overall risk of bias [33]. Differences were resolved with discussion, and if needed, a third reviewer was involved (SL, JST). Study authors were contacted for additional data by e-mail (with a reminder in case of no response) (Additional file 1: eAppendix-4).

\section{Outcomes}

The co-primary outcomes were the change in core symptoms measured with validated rating scales: (1) socialcommunication difficulties (SCD, e.g., ABC-L/SW [34] 
or VABS-Socialization [35]), (2) repetitive behaviors (RB, e.g., ABC-S [34] or CYBOCS-PDD [36]), and (3) overall core symptoms (OCS, e.g., SRS [37] or CARS [38]). There is yet no optimal outcome measure [39], and we accepted a wide range of validated scales, giving preference to clinician-ratings and to the commonly used scales mentioned above, similar to our previous review [4] (Additional file 1: eAppendix-5.3).

Secondary outcomes were premature discontinuation (dropout) due to any reason and due to adverse events, number of participants with a positive response (preferably defined with a CGI-Improvement score $\leq 2$ or at least "much improved" [40]), change in irritability/aggression, ADHD symptoms and anxiety/depression, quality of life, global functioning and caregiver stress (Additional file 1: eAppendix-5.3). We also examined the number of participants with adverse events, sedation, weight gain (preferably defined as $\geq 7 \%$ increase) and extrapyramidal symptoms.

\section{Data analysis}

Random-effects pairwise and network meta-analyses were conducted within a frequentist framework using meta v4.15-1 [41] and netmeta v1.2-1 [42] in R statistical software v4.0.3 [43]. The certainty of evidence of comparisons with placebo for the co-primary outcomes was evaluated using CINeMA (Confidence in Network MetaAnalysis) [44, 45] (Additional file 1: eAppendix-6.9).

The effect-sizes for continuous outcomes were standardized mean differences (SMD, Hedge's g) and for dichotomous outcomes were odds ratios (OR), presented with their 95\% confidence intervals (95\% CI). We post hoc used ORs instead of relative risks, due to their preferred mathematical properties in metaanalysis [46, 47]. In order to present both continuous and dichotomous outcomes in figures, ORs were also converted to SMDs [25]. Treatments were ranked with $P$-scores [48]. Intention-to-treat data were used, whenever available, and methods that handle missing data were preferred to completers' data, giving preference to mixed-models for repeated measures (MMRM) and multiple imputation over last-observation carried forward (LOCF). For dichotomous outcomes, we assumed that participants lost to follow-up did not have a response. The number of participants with a positive response (CGI-Improvement $\leq 2$ ) [40] and weight gain ( $\geq 7 \%$ increase) was imputed from means and standard deviations (SD) using a validated method, when dichotomous data were not reported [49, 50]. Missing SDs were calculated from available statistics [25], pooling subscales (e.g., SRS subscales, assuming a correlation of 0.5) [51] or using the mean SD of included studies [25]. Change scores were preferred to follow-up scores, and the former were estimated post hoc when both baseline and follow-up scores were available using a correlation of 0.5 [25], since baseline imbalances could have inflated treatment effects (Additional file 1: eAppendix-6.1).

RCTs in children/adolescents and adults (or mixed populations) were analyzed separately, since extrapolation between age groups is discouraged [52]. Transitivity was further assessed by comparing the distribution of clinical and methodological variables (i.e., study duration, type of rater, associated conditions at baseline, baseline scores of CGI-Severity (ranging 1-7) [40], ABC-Irritability (ranging 0-45) [34] and mean age). Trials focused on subgroups, i.e., intellectual disability/high-functioning, genetic syndrome or another associated condition, were classified in CINeMA with moderate indirectness [44].

A common heterogeneity variance $\left(\tau^{2}\right)$ was assumed for all comparisons per network, and heterogeneity was quantified as low, moderate or high by comparing $\tau^{2}$ with its empirical distributions [53, 54]. Incoherence was examined globally with a design-by-treatment interaction test and locally with separating indirect from direct evidence [55].

We aimed to include unpublished trials (e.g., contacting authors, using data reported in trial registries, abstracts and reviews), and eligible studies with no usable data were considered in the assessment of reporting bias [44]. Additionally, small-study effects were examined with comparison-adjusted [56] (assuming the direction of bias towards newer medications) and contour-enhanced funnel plots, when there were more than ten studies per comparison [25].

The robustness of the results was investigated in sensitivity analyses using (a) fixed-effects models, excluding studies with (b) implied randomization, (c) genetic syndrome or (d) associated symptoms as inclusion criteria, (e) using only diagnostic evaluation tools, (f) with nonclinician-ratings, (g) from less developed countries (post hoc) [57], (h) with imputed SDs, (i) overall high risk of bias, (j) unclear risk of bias in random sequence generation or allocation concealment (post hoc), (k) open or single-blind, (l) shorter than four weeks, (m) presenting only completers' data, (n) using a correlation of 0.25 and 0.75 to calculate the SD of change scores, and (o) using $\mathrm{ABC}-\mathrm{L} / \mathrm{SW}$ or $\mathrm{ABC}-\mathrm{S}$ (post hoc). In a post hoc sensitivity analysis, relative risks were used for dichotomous outcomes. Baseline severity could not be assessed in a subgroup or sensitivity analysis, due to inconsistent reporting and diversity of scales (Additional file 1: eAppendix-2.2).

Alpha was set at two-sided 0.05, except for heterogeneity, incoherence and funnel plot tests at 0.1 due to their small statistical power. 


\section{Results}

\section{Description of included studies}

Study selection is presented with a PRISMA flow diagram (Additional file 1: eAppendix-4.1), and the list of included/excluded full-texts in Additional file 1: eAppendix-4.2/4.3. From 203 eligible trials, 125 trials in children/adolescents ( $n=7450$ participants) and 18 in adults $(n=1104)$ were included in the quantitative analysis.

Study characteristics are presented in Additional file 1: eAppendix-5.1 and the distribution of potential effect-modifiers in Additional file 1: eAppendix-6.1. The majority of trials were double-blind ( $k=138$ studies), placebo-controlled $(k=137)$ with a parallel-design $(k=110)$ and two-arms $(k=125)$. They were recently published (median publication year of 2015, interquartile range [2008-2019]), had a short duration (12 [8-13] weeks), small sample sizes (40 [23-76]) and few sites (1 [1-3]), which were mainly academic $(k=102$ trials had only academic sites).

The median age of participants was 8.2 [6.3-9.5] years in children/adolescents and 24.6 [21.9-27.9] years in adults. The overall male-to-female ratio was 5.3 [3.9-8.2]. Standardized diagnostic criteria were used in most of the studies (95\%), and seven studies used only diagnostic evaluation tools. Associated symptoms were required as an inclusion criterion in about a third of the studies, mainly irritability and ADHD symptoms (in 30 trials), and a genetic syndrome (neurofibromatosis-type-I) in one trial [58]. At baseline, the sample was moderately to markedly ill with a CGI-S score of 4.8 [4.4-5.1], and ABC-Irritability of 16.9 [13.3-22.3], and about half of the participants had intellectual disability (50\% [0-73.5\%]). Nevertheless, reporting of participant characteristics was poor in about two thirds of the studies.

Risk of bias assessment is presented in Additional file 1: eAppendix-5.2. About 25\% of the studies had an overall low risk of bias, $55 \%$ had moderate and $17 \%$ high. About half adequately reported methods of random sequence and allocation concealment, and blinding was adequately addressed in about $65 \%$. High risk of bias was assigned in about $26 \%$ studies for incomplete outcome data, $36 \%$ for selective reporting and about $12 \%$ for other biases, mainly due to baseline imbalance or early trial termination. Finally, about $30 \%$ of the studies were funded by industry or their investigators applied for a patent.

Forty-one drugs were investigated in 100 trials (antipsychotics and antidepressants in about a third) and 17 dietary-supplements in 43 trials (Additional file 1: eAppendix-5.1). Interventions were connected in mainly star-shaped networks with placebo as the main node (Additional file 2: Fig. S1). Therefore, we focused on comparisons with placebo (Fig. 1, Additional file 3: Fig. S2),

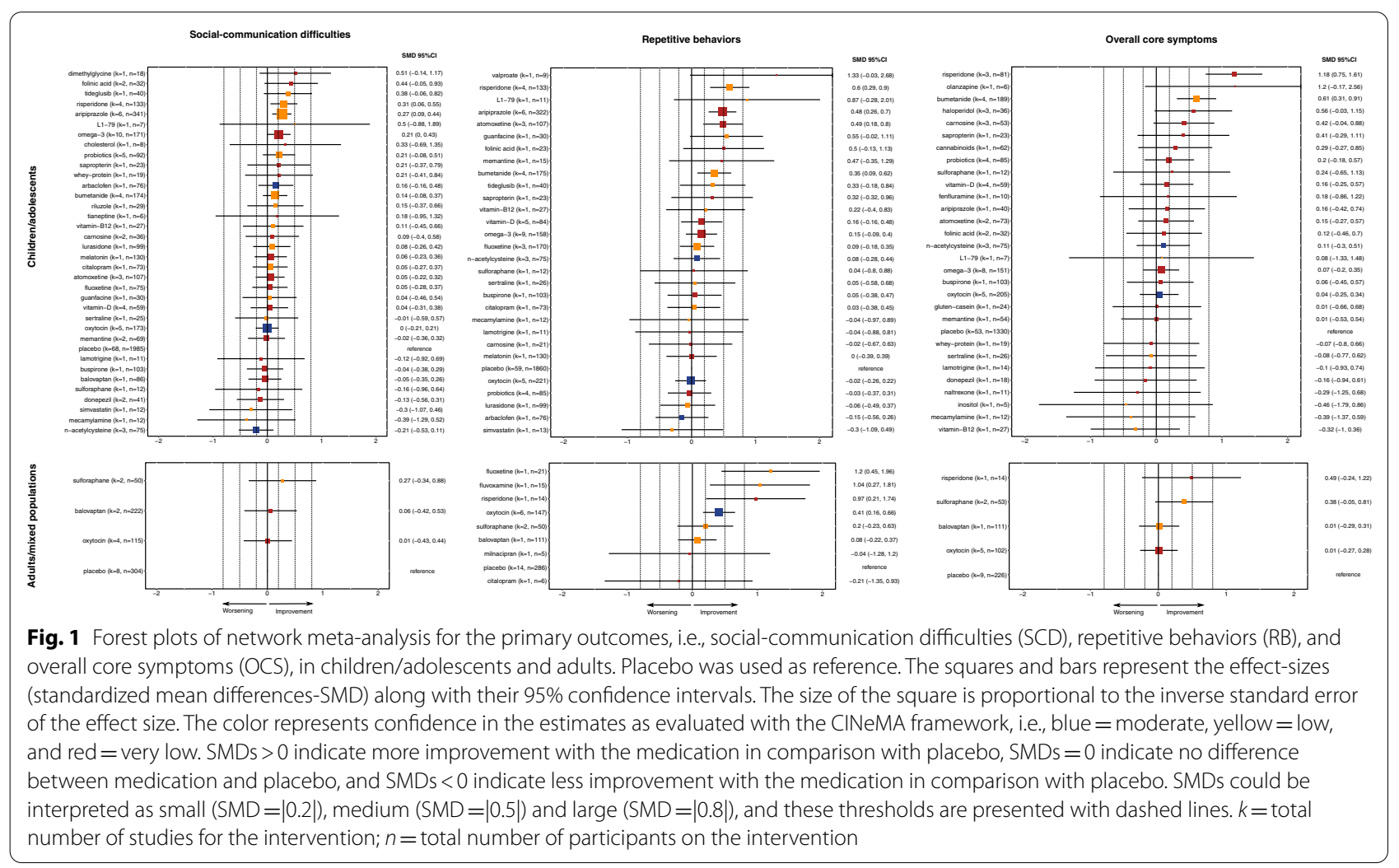


and league tables with all comparisons are presented in Additional file 4: Table S1. The results of pairwise metaanalyses and individual studies are presented in Additional file 5: Fig. S3. In addition, incoherence could not be evaluated when there were no closed loops (i.e., networks for anxiety/depression, quality of life, caregiver stress and all networks in adults). There was no clear indication of incoherence for the rest of the networks, except for irritability, response, weight gain and sedation in children/ adolescents for which pairwise meta-analyses were conducted (Additional file 1: eAppendix-6.8).

\section{Primary outcomes}

\section{Social-communication difficulties (SCD)}

Social-communication difficulties were measured mainly with ABC-L/SW (55\%) and VABS-S (18\%).

In children/adolescents, social-communication difficulties were improved by risperidone $(k=4$ studies in the analysis, $n=133$ participants treated with risperidone; $\mathrm{SMD}=0.3195 \% \mathrm{CI}[0.06,0.55]$; low quality of evidence) and aripiprazole $(k=6, n=341 ; \mathrm{SMD}=0.27[0.09,0.44]$; low). Some trends of improvement were noted for folinic acid $(k=2, n=32, \mathrm{SMD}=0.44[-0.05,0.93]$; very low $)$, tideglusib $(k=1, n=40 ; \mathrm{SMD}=0.38$ [-0.06, 0.82]; low), omega-3-fatty-acids $(k=10, n=171 ; \mathrm{SMD}=0.21[0.00$, $0.43]$, very low), probiotics $(k=5, n=92 ; \mathrm{SMD}=0.21$ $[-0.08,0.51] ; \quad l o w)$ and bumetanide $(k=4, n=174$; $\mathrm{SMD}=0.14[-0.08,0.37]$; low). There were no clear differences between other medications and placebo with very low-to-moderate confidence. Heterogeneity was low $\left(\tau^{2}=0\right)$.

In adults, none of the investigated medications (sulforaphane, balovaptan, oxytocin) improved social-communication difficulties with very-low- or lowquality evidence. There were high levels of heterogeneity $\left(\tau^{2}=0.096\right)$.

\section{Repetitive behaviors (RB)}

Repetitive behaviors were measured mainly with $\mathrm{ABC}-\mathrm{S}$ (47\%) and YBOCS-versions (27\%).

In children/adolescents, repetitive behaviors were improved by risperidone $(k=4, \mathrm{n}=133 ; \mathrm{SMD}=0.60$ $[0.29,0.90]$; low $)$, aripiprazole $(k=6, n=322 ; \mathrm{SMD}=0.48$ $[0.26,0.70]$; very low $)$, atomoxetine $(k=3, n=107$; $\mathrm{SMD}=0.49[0.18,0.80]$; very low $)$ and bumetanide $(k=4$, $n=175 ; \mathrm{SMD}=0.35[0.09,0.62]$, low). There were trends for valproate $(k=1, n=9 ; \mathrm{SMD}=1.33[-0.03,2.68]$; very low) and guanfacine $(k=1, n=30 ; \mathrm{SMD}=0.55[-0.02$, $1.11]$; low), and no clear differences for other medications with very low-to-moderate confidence. Heterogeneity was low-to-moderate $\left(\tau^{2}=0.017\right)$.

In adults, repetitive behaviors were improved by fluoxetine $(k=1, n=21 ; \mathrm{SMD}=1.20[0.45,1.96]$; low $)$, fluvoxamine $(k=1, n=15 ; \mathrm{SMD}=1.04[0.27,1.81]$; low $)$, risperidone $(k=1, n=14 ; \mathrm{SMD}=0.97$ [0.21, 1.74]; very low), and oxytocin $(k=6, n=147 ; \mathrm{SMD}=0.41[0.16$, 0.66]; moderate). Sulforaphane, balovaptan, milnacipran and citalopram were not found efficacious with very low or low confidence. Heterogeneity was low $\left(\tau^{2}=0\right)$.

\section{Overall core symptoms (OCS)}

Overall core symptoms were measured mainly with SRS (47\%) and CARS (22\%).

In children/adolescents, overall core symptoms were improved by risperidone $(k=3, n=81 ; \mathrm{SMD}=1.18$ $[0.75,1.61]$; very low $)$, and bumetanide $(k=4, n=189$; $\mathrm{SMD}=0.61[0.31,0.91] ;$ low $)$. There were some trends for haloperidol $(k=3, n=36$; $\mathrm{SMD}=0.56[-0.03,1.15]$; very low) and carnosine $(k=3, n=53 ; \mathrm{SMD}=0.42[-0.04$, 0.88 ]; very low), and no clear differences for other medications with very low-to-moderate confidence. There were moderate levels of heterogeneity $\left(\tau^{2}=0.038\right)$ and no indication of incoherence. Nevertheless, a small study $(n=30)$ [59] that found no difference between risperidone and memantine $(\mathrm{SMD}=0.00[-0.71,0.72])$ introduced incoherence and was excluded from the primary analysis of this outcome (Additional file 1: eAppendix-6.8), and the results were robust after inclusion of this study (Additional file 6: Fig. S4).

In adults, none of the investigated medications (risperidone, sulforaphane, balovaptan and oxytocin) found to be more efficacious than placebo in reducing overall core symptoms, though a trend was noted for sulforaphane $(k=2, n=53 ; \mathrm{SMD}=0.38[-0.05,0.81] ;$ low $)$. Confidence in evidence was very low or low. Heterogeneity was low $\left(\tau^{2}=0\right)$.

\section{Sensitivity analysis}

The results did not materially change in sensitivity analyses (Additional file 1: eAppendix-6.6, Additional file 6: Fig. S4). There were some potential differences in omega3-fatty-acids. Omega-3-fatty-acids did not reduce socialcommunication difficulties in children/adolescents when studies on associated symptoms were excluded $(k=6$, $n=112, \mathrm{SMD}=0.05[-0.21,0.32])$ or when clinician-ratings were used $(k=3, n=53, \mathrm{SMD}=0.03[-0.36,0.42])$. Yet, their effect-size was larger when $\mathrm{ABC}-\mathrm{L} / \mathrm{SW}$ was used $(k=6, n=79, \mathrm{SMD}=0.45[0.13,0.77])$. In addition, the results for some interventions, i.e., folinic acid, carnosine, vitamin-D, were not robust in sensitivity analyses, which were based on one or two small trials with potentially inflated effect-sizes.

\section{Small-study effects and publications}

There was asymmetry in funnel plots for social-communication difficulties in children/adolescents, indicating 
small-study effects (Additional file 1: eAppendix-6.8). Funnel plots for the other co-primary outcomes were inconclusive. Reporting bias was suspected for some medications, and quality of evidence was downgraded accordingly (Additional file 1: eAppendix-6.9).

\section{Secondary outcomes Irritability}

Irritability was measured mainly with ABC-I (83\%).

In children/adolescents, there was evidence of incoherence (none of the closed loops were incoherent, but $p$-design-by-treatment $=0.014)$ and pairwise meta-analysis were conducted. Irritability was improved by risperidone ( $k=4$ studies in the analysis, $n=138$ participants treated with risperidone; $\mathrm{SMD}=1.05[0.76,1.33]$, $\left.\tau^{2}=0.02\right)$, sulforaphane $(k=1, n=12 ; \mathrm{SMD}=0.97[0.12$, $1.83])$, aripiprazole $(k=5, n=312 ; \mathrm{SMD}=0.63[0.44$, 0.82 ], $\left.\tau^{2}=0\right)$, and citalopram $(k=1, n=73 ; \mathrm{SMD}=0.37$ $[0.04,0.69])$, as well as there was a trend for guanfacine $(k=1, n=30 ; \mathrm{SMD}=0.50[0.00,1.01])$ and riluzole $(k=1$, $n=29 ; \mathrm{SMD}=0.43[-0.09,0.95])$. On the other hand, irritability was worsened by vitamin-B12 $(k=1, n=27$; $\mathrm{SMD}=-0.62[-1.19,-0.05])$ and levetiracetam $(k=1$, $n=10 ; \mathrm{SMD}=-1.47[-2.48,-0.46])$.

In adults, risperidone was found efficacious $(k=1$, $n=14 ; \mathrm{SMD}=1.19[0.34,2.04])$, and heterogeneity was moderate $\left(\tau^{2}=0.028\right)$.

\section{ADHD symptoms}

ADHD symptoms were measured in the majority of the studies with $\mathrm{ABC}-\mathrm{H}$ (79\%).

In children/adolescents, ADHD symptoms were improved by olanzapine $(k=1, n=6 ; \mathrm{SMD}=2.08[0.48$, 3.68], based only on indirect evidence), guanfacine $(k=1$, $n=30 ; \mathrm{SMD}=1.39[0.73,2.05])$, aripiprazole $(k=7$, $n=363 ; \mathrm{SMD}=0.82[0.59,1.05])$, risperidone $(k=5$, $n=155 ; \mathrm{SMD}=0.79 \quad[0.47,1.11])$, naltrexone $(k=1$, $n=23 ; \mathrm{SMD}=0.85[0.12,1.59])$, and atomoxetine $(k=3$, $n=107$; SMD $=0.64[0.30,0.99])$, as well as a trend was noted for sulforaphane $(k=1, n=12$; SMD $=0.88[-0.03$, $1.80])$. Heterogeneity was moderate $\left(\tau^{2}=0.032\right)$.

In adults, none of the investigated medications were found efficacious for ADHD symptoms, and heterogeneity was low $\left(\tau^{2}=0\right)$.

\section{Anxiety/depressive symptoms}

Different scales measured anxiety/depression in children/ adolescents (e.g., CBCL-I, BASC-I, CASI, DBC-Anxiety), and STAI-state was used in half of the studies in adults. None of the investigated medications found to improve anxiety or depressive symptoms, except for a trend about risperidone in adults $(n=1, k=14 ; \mathrm{SMD}=0.67$ $[-0.07,1.41])$. There were moderate-to-high levels of heterogeneity in children/adolescents $\left(\tau^{2}=0.041\right)$ and low in adults $\left(\tau^{2}=0\right)$.

\section{Caregiver stress}

Caregiver stress was measured mainly with PSI (36\%), CSQ (22\%) and CGSQ (14\%) in children/adolescents, and with PedsQL-Family Impact in adults. In children/adolescents, it was reduced by melatonin $(k=1$, $n=54 ; \mathrm{SMD}=0.51[0.12,0.91])$, and there were trends of small improvements by cannabinoids $(k=1, n=80$; $\mathrm{SMD}=0.32[-0.06,0.69])$ and atomoxetine $(k=3$, $n=104 ; \mathrm{SMD}=0.21[-0.06,0.48])$. There were no clear differences between other medications and placebo in both age groups, and heterogeneity was low $\left(\tau^{2}=0\right)$.

\section{Global functioning}

Global functioning was measured with GAF or CGAS. In children/adolescents, it was improved by risperidone $(k=3, n=62, \mathrm{SMD}=0.83[0.40,1.26])$ and aripiprazole $(k=2, n=69, \mathrm{SMD}=0.75[0.33,1.17])$. No clear differences between other investigated medications and placebo were found in both age groups. Heterogeneity was moderate in children/adolescents $\left(\tau^{2}=0.016\right)$ and low in adults $\left(\tau^{2}=0\right)$.

\section{Quality of life}

Quality of life was measured with PedsQL in children/ adolescents, and with PedsQL (40\%) and WHO-QOL $(60 \%)$ in adults. There were no clear differences between medications and placebo in children/adolescents. In adults, quality of life was improved by balovaptan $(k=2$, $n=217 ; \mathrm{SMD}=0.22[0.02,0.43])$, and potentially by oxytocin $(k=3, n=41$; SMD $=0.44[-0.02,0.90])$. Heterogeneity was low in both age groups $\left(\tau^{2}=0\right)$.

\section{Response}

Pairwise meta-analyses were conducted in children/adolescents due to incoherence ( $50 \%$ of the closed loops were incoherent; $p$-design-by-treatment $=0.068)$. In comparison with placebo, more participants responded with risperidone $\left(k=5, n=161 ; \mathrm{OR}=11.33[4.99,25.70] ; \tau^{2}=0.294\right)$, guanfacine $(k=1, n=30 ; \mathrm{OR}=9.67[2.41,38.71])$, wheyprotein $(k=1, n=22 ; \mathrm{OR}=4.56[1.25,16.63])$, aripiprazole $\left(k=5, n=317 ; \mathrm{OR}=4.26[2.32,7.83] ; \tau^{2}=0.212\right)$, vitamin-B12 $(k=1, n=28 ; \quad \mathrm{OR}=3.83 \quad[1.20,12.28])$, atomoxetine $(k=3, n=109 ; \quad \mathrm{OR}=3.18 \quad[1.56,6.48]$; $\left.\tau^{2}=0\right)$, melatonin $(k=1, n=60 ; \mathrm{OR}=3.06[1.38,6.77])$, bumetanide $\left(k=3, n=155 ; \mathrm{OR}=2.78[1.48,5.21] ; \tau^{2}=0\right)$, and cannabinoids $(k=1, n=100 ; \mathrm{OR}=2.56[1.15,5.70])$, while fewer with oral human immunoglobulins (IGOH) $(k=1, n=94 ; \mathrm{OR}=0.40[0.16,0.99])$. There were no clear differences for other medications. 
In adults, there were more responders with risperidone $(k=1, n=15 ; \mathrm{OR}=37.40[1.62,865.22])$ and fluvoxamine $(k=1, n=15 ; \mathrm{OR}=35.13[1.52,814.72]$. There were high levels of heterogeneity $\left(\tau^{2}=0.257\right)$.

\section{Dropouts due to any cause}

In children/adolescents, fewer overall dropouts were noted with risperidone $(k=10, n=274 ; \mathrm{OR}=0.38[0.22$, $0.65])$, lurasidone $(k=1, n=100 ; \mathrm{OR}=0.35[0.14,0.88])$ and aripiprazole $(k=8, n=399 ; \mathrm{OR}=0.46[0.29,0.75])$, as well as potentially with melatonin $(k=4, n=239$; $\mathrm{OR}=0.52[0.26,1.03])$. More dropouts were observed with arbaclofen $(k=1, n=76$; OR $=3.39[1.16,9.88])$, and a trend was noted for fluoxetine $(k=3, n=161 ; \mathrm{OR}=1.59$ $[0.97,2.58])$. There were no clear differences for other medications, and there were some indications of incoherence $(12.5 \%$ of the loops were incoherent; $p$-design-bytreatment $=0.334)$. In adults, there were no clear differences for the investigated medications. Heterogeneity was low in both age groups $\left(\tau^{2}=0.006\right.$ and $\left.\tau^{2}=0\right)$.

\section{Dropouts due to adverse events}

There were no clear differences between investigated medications and placebo in both age groups, and heterogeneity was low $\left(\tau^{2}=0\right)$.

\section{Any adverse event}

In children/adolescents, more participants had adverse events with risperidone $(k=4, n=123$; OR $=4.74$ [2.24, 10.04]), citalopram $(k=1, n=73 ; \quad \mathrm{OR}=5.38 \quad[1.14$, 25.46]), fluvoxamine $(k=1, n=18 ; \mathrm{OR}=4.50 \quad$ [1.02, 19.90]) and aripiprazole $(k=6, n=348 ; \mathrm{OR}=2.62[1.65$, $4.15])$, as well as potentially with guanfacine $(k=1$, $n=30$; OR $=17.94[0.98,329.56])$ and lurasidone $(k=1$, $n=100$; OR $=1.92[0.95,3.90])$. In adults, more participants had adverse events with risperidone $(k=1, n=15$; $\mathrm{OR}=14.30[2.19,93.37])$. There were no clear differences between other medications and placebo. Heterogeneity was low in children/adolescents $\left(\tau^{2}=0\right)$ and moderate in adults $\left(\tau^{2}=0.049\right)$.

\section{Sedation}

In children/adolescents, pairwise meta-analyses were conducted due to incoherence ( $75 \%$ of the closed loops were incoherent; $p$-design-by-treatment $=0.051)$. More participants had sedation with guanfacine $(n=1, k=30$; $\mathrm{OR}=62.83[12.84,307.45])$, haloperidol $(n=1, k=20$; $\mathrm{OR}=44.33[4.78,410.96])$, risperidone $(n=4 ; k=142$, $\left.\mathrm{OR}=11.95[5.86,24.36], \tau^{2}=0\right)$, aripiprazole $(n=5$, $\left.k=317 ; \mathrm{OR}=3.56[1.62,7.86] ; \tau^{2}=0\right)$ and melatonin $(n=1, k=60 ; \mathrm{OR}=3.28[1.25,8.59])$.
In adults, there were no clear differences, and heterogeneity was low $\left(\tau^{2}=0\right)$.

\section{Weight gain}

In children/adolescents, there was evidence of incoherence $(50 \%$ of the closed loops were incoherent; $p$-designby-treatment $=0.032$ ) and pairwise meta-analyses were conducted. More participants had weight gain with aripiprazole $\left(n=5, k=317\right.$; OR $\left.=3.78[2.09,6.84], \tau^{2}=0\right)$ and risperidone $(n=5, k=161 ; \mathrm{OR}=3.39$ [1.80, 6.38], $\left.\tau^{2}=0\right)$ in comparison with placebo, while aripiprazole caused less weight gain in comparison with risperidone $\left(n=2, k=104 ; \mathrm{OR}=0.22[0.09,0.55], \tau^{2}=0.045\right)$. There were no clear differences between other medications.

In adults, none of the investigated medications (sulforaphane, oxytocin and balovaptan) was associated with weight gain, and heterogeneity was low $\left(\tau^{2}=0\right)$.

\section{Extrapyramidal symptoms}

The network of children/adolescents was disconnected; therefore, pairwise meta-analyses were conducted. In comparison with placebo, more participants had extrapyramidal symptoms with risperidone $(n=4, k=142$; $\left.\mathrm{OR}=3.02[1.22,7.48] ; \tau^{2}=0\right)$ and aripiprazole $(n=4$, $k=300 ; \mathrm{OR}=2.38$ [1.18, 4.77]; $\tau^{2}=0$ ).

There were no data available for adults.

\section{Discussion}

This is the first comprehensive network meta-analysis on pharmacological and dietary-supplement interventions for ASD. Pediatric and adult populations were analyzed separately, in order to avoid misleading extrapolations [52]. Core symptom domains (SCD and $\mathrm{RB}$ ) were also examined separately as co-primary outcomes, since differential treatment responses can be expected [52]. In addition, scales that measure overall core symptoms (OCS) in single scores were considered as a distinct outcome. Associated symptoms and side-effects were also investigated as secondary outcomes. Therefore, our analysis provides a more comprehensive synthesis of evidence in comparison with previous reviews that were mainly focused on pediatric populations, certain symptoms or specific medications, or did not utilize a network meta-analysis [9-17, 19, 21, 23, 24].

Our review identified the following medications that could improve at least one core symptom domain: aripiprazole ( $\mathrm{SCD}, \mathrm{RB}$ ), atomoxetine (RB), bumetanide ( $\mathrm{RB}, \mathrm{OCS}$ ) and risperidone (SCD, RB, OCS) in children/ adolescents; fluoxetine (RB), fluvoxamine (RB), oxytocin $(\mathrm{RB})$ and risperidone (RB) in adults. In addition, there were some indications of improvement by carnosine, haloperidol, folinic acid, guanfacine, omega-3-fattyacids, probiotics, sulforaphane, tideglusib and valproate, 
yet they were imprecise based on limited data and not formally statistically significant, as well as not robust in sensitivity analysis.

\section{Summary of evidence Commonly used medications}

Currently, no medication is approved for the core symptoms of ASD [39]. However, about half of the individuals with ASD receive psychotropic drugs, mainly for associated symptoms, such as antipsychotics (median prevalence of 18.1\%), ADHD medications (16.6\%), antidepressants (17.2\%), antiepileptics/ mood-stabilizers and sleep medication [8]. Findings of our analysis on these medications are summarized in Fig. 2, facilitating intuitive understanding of the current evidence.

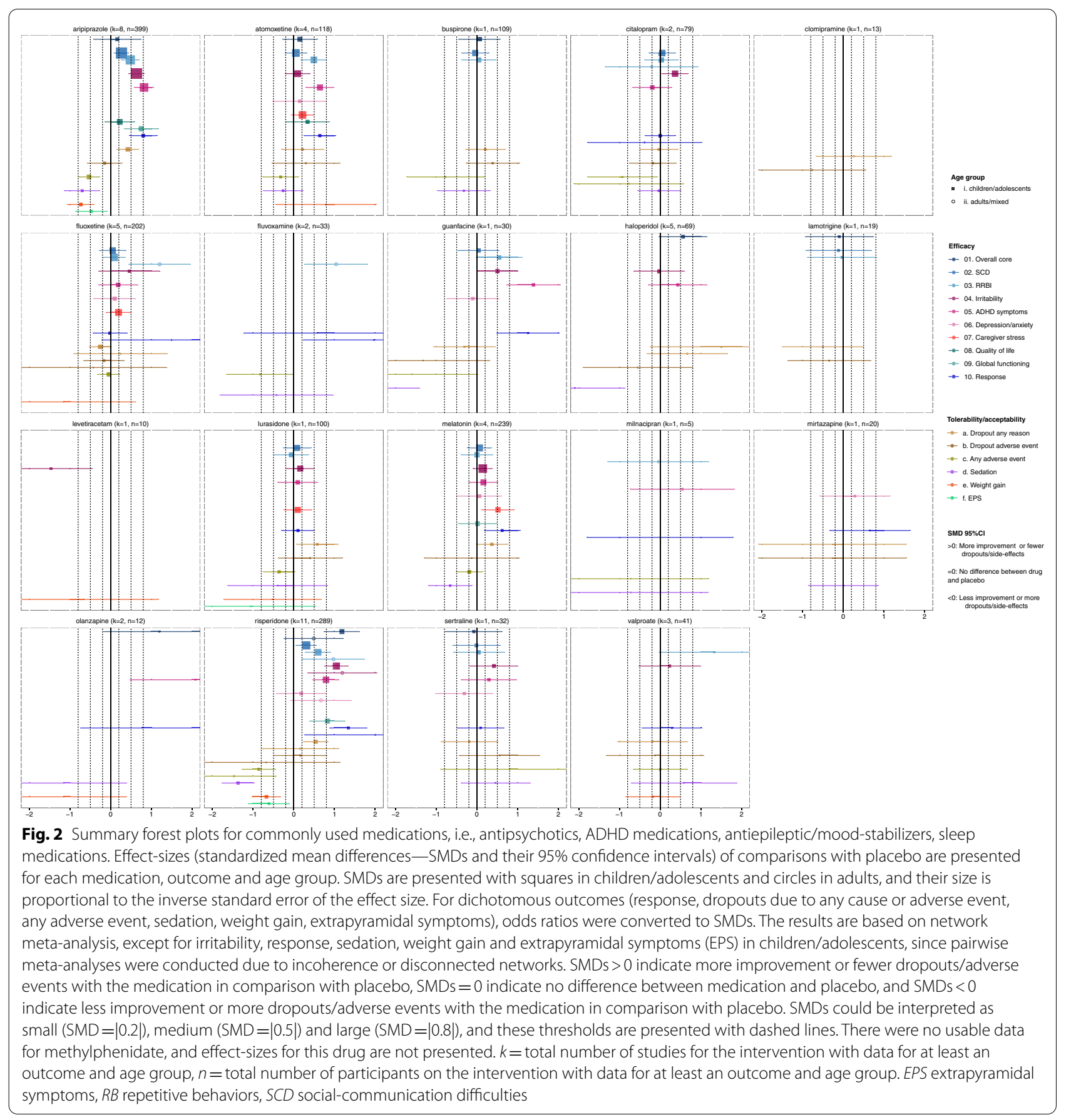


Among antipsychotics, aripiprazole and risperidone demonstrated medium-to-large effect-sizes in reducing irritability and ADHD symptoms, while smaller improvements were found in social-communication difficulties and repetitive behaviors. On the other hand, lurasidone was in general not efficacious, and there were only a few data available for olanzapine and haloperidol, and for adults. Antipsychotics were also associated with more adverse events, sedation, weight gain and extrapyramidal symptoms. Nevertheless, reporting bias was suspected (Additional file 1: eAppendix-6.8), e.g., two pediatric studies found that risperidone did not improve social-communication difficulties as measured with $\mathrm{ABC}-\mathrm{L} / \mathrm{SW}$, yet there were no usable data for this analysis $[60,61]$. In addition, trials on antipsychotics were conducted mainly in participants with irritability. As a result, improvements in core symptoms could be collateral to the reduction in interfering challenging behaviors that can subsequently allow participation in social interactions [62]. In other words, antipsychotics may not have direct effects on core symptoms, but rather secondary to the reduction in irritability. Trials focusing on core symptoms are sparse, and data from a small trial $(n=41)$ investigating risperidone for repetitive behaviors are not yet reported [63]. Therefore, evidence was downrated due to indirectness and reporting bias (Additional file 1: eAppendix-6.9).

Among ADHD medications, atomoxetine and guanfacine improved ADHD symptoms and potentially repetitive behaviors, but not social-communication difficulties. Guanfacine was also associated with more adverse events and sedation. A causal-mediation analysis suggested a causal link from hyperactivity to repetitive behaviors and from impulsivity/inattention to social-communication difficulties in ASD [64]. Therefore, and since these drugs were investigated in participants with ADHD symptoms, improvements in repetitive behaviors could be indirect and subsequent to the reduction in hyperactivity. Of note, there were no usable data for methylphenidate, since none of the five crossover trials reported usable data from the first phase (Additional file 1: eAppendix-6.8), and none of the ADHD medications were investigated in adults.

Antidepressants and buspirone were not found efficacious for core or associated symptoms in children/adolescents, except citalopram that improved irritability with a small-to-medium effect-size. Citalopram, fluvoxamine and fluoxetine were also associated with more adverse events or dropouts. In adults, however, fluoxetine and fluvoxamine improved repetitive behaviors with large effect-sizes, yet based on single small $(n=30-37)$ studies $[65,66]$. Apart from the limited data for adults, such differences might be explained by different study designs, participant characteristics and age-dependent variability in treatment response $[67,68]$.

Antiepileptics/mood-stabilizers were in general not efficacious based on limited and very low-quality data. A single small study $(n=13)$ suggested efficacy for valproate [69], yet there was reporting bias and two additional studies did not report appropriate data [70, 71] (Additional file 1: eAppendix-6.8). Of note, levetiracetam worsened irritability with a large effect-size in a small study $(n=12)$ [72], in accordance with the well-documented behavioral side-effects of this drug [73]. Last, melatonin was not efficacious for core or associated symptoms, yet it decreased caregiver stress and increased the number of responders. Such beneficial effects could be collateral to the reduction in sleep problems $[2,74,75]$. Sleep outcomes were not investigated in this review, but our findings support its sedative effects.

\section{Experimental medications}

Our review identified a considerable number of experimental medications (Fig. 3) with diverse mechanisms of action, which discussion is out of the scope of this review (e.g., see [39, 76-79]). The majority of them were investigated exclusively in children/adolescents, except for oxytocin and balovaptan.

Oxytocin and balovaptan (vasopressin- $\mathrm{V}_{1 \mathrm{~A}}$ receptor antagonist) were not efficacious in children/adolescents, based on substantial evidence from large trials, e.g., $(n=290-339)$ [80, 81]. In adults, however, oxytocin improved repetitive behaviors with small-to-medium effect-sizes and moderate-quality evidence. This finding needs replication, since studies were mainly focused on high-functioning participants and variability in treatment response due to age-dependent differences in the oxytocin system cannot be excluded $[82,83]$. Balovaptan was not found efficacious in adults based on two large studies $(n=223-322)$ [84, 85], yet small improvements in quality of life were noted. Of note, intranasal vasopressin was efficacious in a small trial $(n=30)$ [86], which was, however, excluded from our analysis due to unconcealed allocation (Additional file 1: eAppendix-4.2.).

Bumetanide (loop-diuretic that may enhance GABAergic inhibition) was found to improve repetitive behaviors and overall core symptoms with small-to-medium effect-sizes, but not social-communication difficulties. However, two large phase-III trials $(n=422$ in total) [87] were negative and prematurely terminated [88], yet they did not report usable data, and therefore, evidence was downrated due to reporting bias. Other experimental medications were not found efficacious based on current data. There were some indications for cannabinoids (more participants had a positive response), and naltrexone (improvement of ADHD symptoms), 


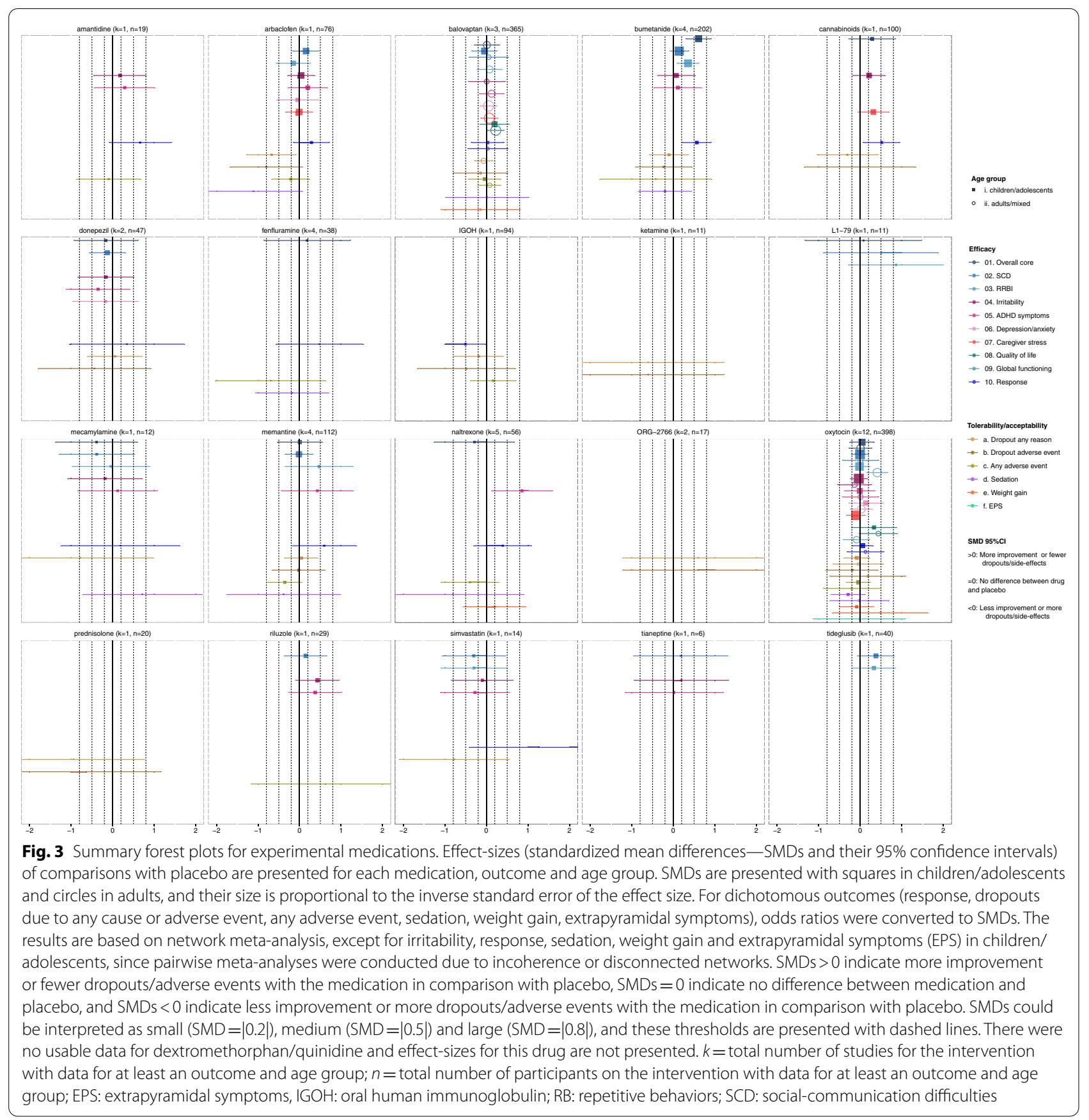

yet they were based on single studies $[89,90]$ and there was also reporting bias for naltrexone (Additional file 1: eAppendix-6.8). On the other hand, arbaclofen $\left(\mathrm{GABA}_{B}\right.$ agonist) was associated with more dropouts and IGOH (oral human immunoglobulin) with fewer responders. Nevertheless, several trials are ongoing, e.g., for arbaclofen [91, 92], memantine [93] and cannabinoids [94, 95]. In addition, the findings on tideglusib (GSK-3 $\beta$ inhibitor), L1-79 (tyrosine hydroxylase inhibitor) and riluzole could be imprecise, since data from abstracts were used [96-98].

\section{Dietary-supplements}

The efficacy of dietary-supplements was inconclusive (Fig. 4). Omega-3-fatty-acids could potentially improve social-communication difficulties with small effect-sizes, based on very low-quality evidence from ten studies in children/adolescents. Similarly, there were some trends 


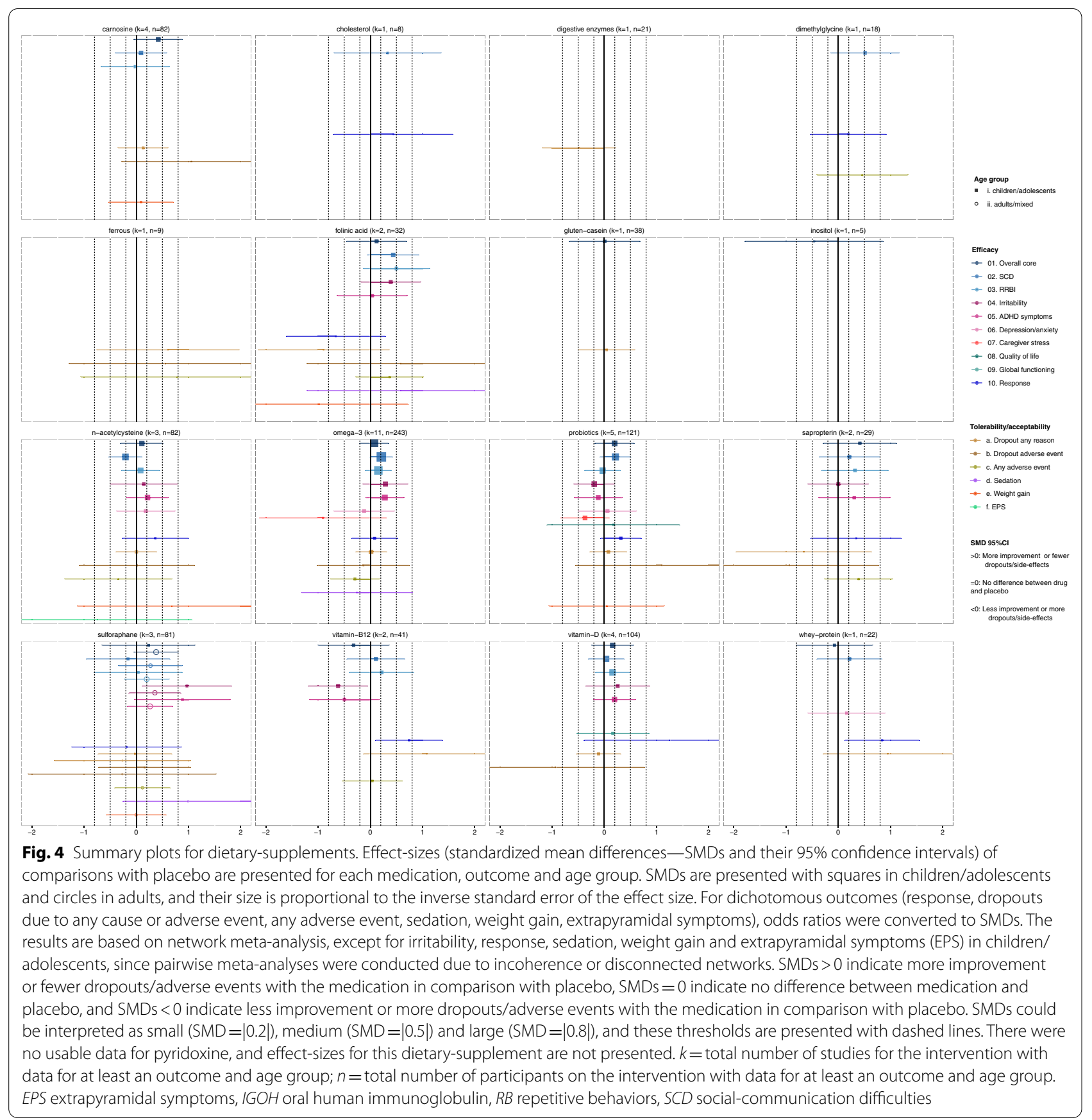

for carnosine, folinic acid and probiotics, based on fewer data. Nevertheless, these findings were highly heterogeneous (for carnosine and folinic acid), imprecise and not statistically significant (at two-sided alpha 0.05), and not robust in sensitivity analyses. Therefore, results from larger trials are warranted, e.g. [99, 100]. There was also mixed evidence about sulforaphane (broccoli sprout extract), since findings were based on one inconclusive trial $(n=45)$ in children/adolescents [101], and two contradicting trials $(n=44-48)$ in adults $[102,103]$, while usable data from a larger trial $(n=110)$ are not yet reported [104]. In addition, there were some indications from single studies for cysteine-rich whey-protein [105] and vitamin-B12 [106], since both increased the number of responders but were not found to be efficacious for core or associated symptoms. On the contrary, vitamin-B12 worsened irritability with a medium effectsize, which is in line with a meta-analysis of prevalence 
that identified its potential behavioral side-effects [107]. Therefore, the safety of dietary-supplements should not be overlooked.

\section{Limitations}

There are certain limitations. First, and in contrast with other fields of psychopharmacology, evidence base of ASD is flooded by small trials focusing on associated symptoms and investigating a plethora of medication classes, for which adequate dosing or duration of treatment is still unclear, and some of them have not yet investigated in RCTs. This reflects the two main approaches that guide psychopharmacology in ASD, by re-purposing psychotropics for associated symptoms or by targeting neurobiological processes [2]. Nevertheless, ASD may not be a unitary diagnosis in terms of neurobiology, according to its heterogeneity and lack of biomarkers. Therefore, it is likely that there is substantial interpersonal variability of treatment response across medications. Individual-participant-data meta-analyses could further explore this issue and investigate the potential impact of participant-level covariates [108], e.g., age, sex, baseline severity of core and associated symptoms. In that direction, there are also efforts to disentangle the neurobiology of subgroups within ASD in order to facilitate biomarker stratification and more targeted treatments $[39,77]$.

Second, clinical trials in ASD could be prone to substantial placebo responses and a lower ability to detect efficacy, which may be increased with adequately powered trials, rigorous selection of participants and careful selection of outcome measures [4]. In line with this, there is lack of consensus on outcome measures [39], and different scales are often used. We accepted a wide range of validated scales in order to incorporate more evidence, yet we preferred recommended and commonly used scales in order to obtain comparable measures (Additional file 1: eAppendix-5.3). As a result, data for most of the outcomes were derived mainly from one or two scales (Additional file 1: eAppendix-5.1), which treatment effects might agree in most cases, e.g., as suggested between CYBOCS and ABC-S [39]. The results were also generally robust in sensitivity analyses when clinician-ratings or when $A B C$ subscales were used, except for some potential differences in omega-3-fattyacids (Additional file 1: eAppendix-6.6, Additional file 6: Fig. S4). Nevertheless, further research is needed, since scales with different psychometric properties, e.g., sensitivity to change or susceptibility to placebo effects, could demonstrate discordant treatment effects. For example, a trial found low-dose buspirone to improve repetitive behaviors as measured with ADOS-RRB and RBS, but not with CYBOCS [109], which was preferred in our analysis according to our hierarchy (Additional file 1: eAppendix-5.3).

Third, there were limited data for adults, some medications, e.g., methylphenidate, and secondary outcomes, e.g., anxiety/depressive symptoms, which are, however, considered one of the top research priorities [110,111]. Fourth, our analysis was mainly based on star-shaped networks of placebo-controlled comparisons and only a few medications were investigated in more than one or two trials, often with small sample sizes. Therefore, heterogeneity and incoherence could be masked, due to the low statistical power of their tests. Small-study effects could also be masked, since comparison-adjusted funnel plots should be interpreted with great caution when there are a few trials per comparison. Fifth, transitivity assumption could not be adequately assessed, since effect-modifiers are still unclear and insufficiently reported in clinical trials. Therefore, and despite of ordering treatments by their ranking in forest plots, indirect evidence, treatment hierarchies and league tables should be interpreted with great caution. There was also evidence of incoherence in irritability, response, sedation and weight gain in children/adolescents; therefore, pairwise meta-analyses were conducted for these outcomes. In addition, about half of the studies stated to be randomized without an exact description of the randomization method, yet the results did not materially change in sensitivity analyses when studies with an unclear risk of bias in random sequence generation or allocation concealment were excluded (Additional file 1: eAppendix-6.6, Additional file 6: Fig. S4).

Last, a comprehensive review of tolerability was beyond the scope of the manuscript, yet we examined dropouts and important side-effects that overlap among drug classes, i.e., sedation, weight gain and extrapyramidal symptoms, and our findings are in line with the literature [112]. Nevertheless, medications with different mechanisms of action can have unique side-effect profiles, e.g., bumetanide as a loop-diuretic can cause diuresis and hypokalemia [113]. Individuals with ASD may also be more sensitive to side-effects in comparison with neurotypical individuals [2]. Therefore, medications should be used after careful consideration and monitoring of their safety [2], as well as at low doses, since a therapeutic window could be expected, e.g., for risperidone [114].

\section{Conclusions}

In conclusion, there was evidence that some medications could improve social-communication difficulties and/ or repetitive behaviors in children/adolescents: aripiprazole, atomoxetine, bumetanide, and risperidone; while some medications could improve repetitive behaviors in adults: fluoxetine, fluvoxamine, oxytocin and risperidone. 
A large part of the evidence consisted of small RCTs (median 40 participants) with a short duration (median 12 weeks) and limited generalizability. Therefore, current commonly used medications, i.e., antipsychotics and ADHD medications, can be used for associated symptoms as indicated, and smaller improvements in core symptoms could also be expected, at least collaterally to the improvement of challenging behaviors. These medications are associated with side-effects, and therefore, they should be prescribed only after careful consideration and monitoring of their benefit-risk ratio. Evidence on the efficacy and safety for other medications, including bumetanide, oxytocin and some dietary-supplements, is at best preliminary and warrants further investigation. In line with the limitations of our review, there are current efforts to advance clinical psychopharmacology in ASD (e.g., within the AIMS-2-Trials consortium or the ISCTM/ECNP ASD working group), first with the elucidation of its neurobiology and the development of more targeted medications, second with the use of appropriate scales for measuring core symptoms, and third with welldesigned and adequately powered clinical trials $[39,77]$.

\begin{abstract}
Abbreviations
95\% Cl: 95\% Confidence intervals; ASD: Autism spectrum disorder; ABC-H: Aberrant Behavior Checklist-Hyperactivity/noncompliance; ABC-I: Aberrant Behavior Checklist-Irritability; ABC-L/SW: Aberrant Behavior Checklist-Lethargy/Social-Withdrawal; ABC-S: Aberrant Behavior Checklist-Stereotypic behavior; ADHD: Attention Deficit Hyperactivity Disorder; ADI-R: Autism Diagnostic Interview-Revised; ADOS-RRB: Autism Diagnostic Observation Schedule-Restricted and Repetitive Behaviors; BASC-I: Behavior Assessment System for Children - Internalizing; CARS: Childhood Autism Rating Scale; CASI: Childhood Anxiety Sensitivity Index; CBCL-I: Child Behavior ChecklistInternalizing; CGAS: Children Global Assessment Scale; CGl: Clinical Global Impression; CGSQ: Caregiver Strain Questionnaire; CINeMA: Confidence in Network Meta-Analysis; CSQ: Client Satisfaction Questionnaire; CYBOCS(-PDD): Children's Yale-Brown Obsessive Compulsive Scale (Modified for Pervasive Developmental Disorders); DBC-Anxiety: Developmental Behavior Checklist-Anxiety; DSM: Diagnostic and Statistical Manual of Mental Disorders; GAF: Global Assessment of Functioning; FDA: Food and Drug Administration; ICD: International Classification of Diseases; IGOH: Oral human immunoglobulin; IQR: Interquartile range; OCS: Overall core symptoms; OR: Odds ratio; LOCF: Last-observation carried forward; MMRM: Mixed-models for repeated measures; PedsQL: Pediatric Quality of Life Inventory; PSI: Parental Stress Index; RB: Repetitive Behaviors; RBS: Repetitive Behavior Scale; RCT: Randomized Controlled Trials; SCD: Social Communication difficulties; SD: Standard deviation; SMD: Standardized Mean Differences; SRS: Social Responsiveness Scale; STAI: State-Trait Anxiety Inventory; VABS: Vineland Adaptive Behavior Scale; WHO-QoL: World Health Organization-Quality of Life.
\end{abstract}

\section{Supplementary Information}

The online version contains supplementary material available at https://doi. org/10.1186/s13229-022-00488-4.

Additional file 1. eAppendix.

Additional file 2. Fig. S1. Network plots.

Additional file 3. Fig. S2. Forest plots for secondary outcomes.

Additional file 4. Table S1. League tables.
Additional file 5. Fig. S3. Forest plots for pairwise meta-analysis and individual studies.

Additional file 6. Fig. S4. Sensitivity analyses.

Additional file 7. Dataset.

\section{Acknowledgements}

We would like to thank Farhad Shokraneh, former information specialist of the Cochrane Schizophrenia Group, who conducted the first search in electronic databases, Dr. Yikang Zhu for the translation of a Chinese study and Prof. Toshi Furukawa for the translation of a Japanese study. We would like to thank the following authors that contributed to the review by kindly providing additional data and/or clarifications regarding their studies: Adi Aran, Kaat Alaerts, Nadir Aliyev, Eugene Arnold, Haim Belmaker, Yéhézkel Ben-Ari, Leventhal Bennet, Stephen Bent, Helena Brentani, Jan Buitelaar, Ana Maria Castejon, Michael Chez, Torsten Danfors, Paulo Fontoura, Robert Grimaldi, Paul Gringras, Alexander Häge, Randi J. Hagerman, Antonio Hardan, Robert Hendren, Janet Kern, Bruno Leheup, Wenn Liu, Raquel Martinez, James McCracken, Tali Nir, Deborah Pearson, Laura Politte, Jeanette Ramer, Dan Rossignol, Kevin Sanders, Elisa Santocchi, Renato Scifo, Sarah Shea, Lawrence Scahill, Jeremy VeenstraVanderweele Paul Wang, David Wilensky, Hidenori Yamasue, and Lingli Zhang.

\section{Authors' contributions}

SS contributed to study design, study selection, data extraction, contacting authors for additional data, statistical analysis, interpretation of the data, drafting the first version of the manuscript, and study supervision; OC contributed to study selection, data extraction, and contacting authors for additional data; HW contributed to study selection, data extraction, and contacting authors for additional data; JST contributed to data extraction and technical support with Access database; IB contributed to study selection; MK contributed to study selection; AR contributed to data extraction; AC contributed to study selection; GD contributed to study selection; MH contributed to technical support with Access database; DF contributed to study design and interpretation of the data; AJCS contributed to interpretation of the data; DM contributed to statistical analysis and interpretation of the data; TC contributed to interpretation of the data; DGM contributed to interpretation of the data; MP contributed to study design and interpretation of the data; CA contributed to study design and interpretation of the data; SL contributed to study design, data extraction, statistical analysis, interpretation of the data, drafting the first version of the manuscript, and study supervision. All authors critically reviewed the manuscript for important intellectual content. The authors read and approved the final manuscript.

\section{Funding}

This project has received funding from the Innovative Medicines Initiative 2 Joint Undertaking under Grant Agreement No. 777394 for the project AIMS2-TRIALS. This Joint Undertaking receives support from the European Union's Horizon 2020 research and innovation programme and EFPIA and AUTISM SPEAKS, Autistica, SFARI. CA, MP and DF were supported by the Spanish Ministry of Science, Innovation and Universities. Instituto de Salud Carlos III, co-financed by ERDF Funds from the European Commission, "A way of making Europe", CIBERSAM, Madrid Regional Government (B2017/BMD-3740 AGES-CM-2), European Union Structural Funds and European Union Seventh Framework Program and H2020 Program; Fundación Familia Alonso, Fundación Alicia Koplowitz and Fundación Mutua Madrileña. The funders had no role in the design of the study; in the collection, analyses, or interpretation of data; in the writing of the manuscript, or in the decision to publish the results. Any views expressed are those of the authors and not necessarily those of the funders.

Availability of data and materials

The dataset used in the current study is available in this published article and its Additional file 7: Dataset.

\section{Declarations}

Ethics approval and consent to participate

Not applicable. 


\section{Consent for publication}

Not applicable.

\section{Competing interests}

In the past 3 years Stefan Leucht has received honoraria as a consultant and/ or advisor and/or for lectures from Alkermes, Angelini, Eisai, Gedeon Richter, Janssen, Lundbeck, Lundbeck Institute, Merck Sharpp and Dome, Otsuka, Recordati, Rovi, Sanofi Aventis, TEVA, Medichem, Mitshubishi. David Fraguas has been a consultant and/or has received fees from Angelini, Casen, Janssen, Lundbeck, and Otsuka. He has also received grant support from Instituto de Salud Carlos III (Spanish Ministry of Science and Innovation) and from Fundación Alicia Koplowitz.Mara Parellada has received educational honoraria from Otsuka, research grants from FAK and Fundación Mutua Madrileña (FMM), Instituto de Salud Carlos III (Spanish Ministry of Science, Innovation and Universities) and European ERANET and H2O2O calls, travel grants from Otsuka and Janssen. Consultant for Exeltis and Servier. Celso Arango has been a consultant to or has received honoraria or grants from Acadia, Angelini, Gedeon Richter, Janssen Cilag, Lundbeck, Otsuka, Roche, Sage, Sanofi, Servier, Shire, Schering Plough, Sumitomo Dainippon Pharma, Sunovion and Takeda. Maximilian Huhn has received speakers honoraria from Janssen. Declan Murphy has received consulting fees from Roche. Antonia San José Cáceres has been a consultant for Roche and is currently involved in clinical trials with Servier. The other authors have nothing to disclose.

\section{Author details}

${ }^{1}$ Department of Psychiatry and Psychotherapy, School of Medicine, Technical University of Munich, Ismaningerstr. 22, 81675 Munich, Germany. ${ }^{2}$ Department of Child and Adolescent Psychiatry, Mardin State Hospital, Artuklu, Mardin, Turkey. ${ }^{3}$ Department of Psychiatry and Psychotherapy, School of Medicine, Technical University of Munich, Munich, Germany. ${ }^{4}$ Department of Experimental and Clinical Medicine, Psychiatric Clinic University Hospital 'Gaspare Rodolico', University of Catania, Catania, Italy. ${ }^{5}$ Department of Psychiatry, Spedali Civili Hospital, Brescia, Italy. ${ }^{6}$ Department of Psychiatry, Psychosomatic Medicine and Psychotherapy, Social Foundation Bamberg, Teaching Hospital of the University of Erlangen, Bamberg, Germany. Institute of Psychiatry and Mental Health, Hospital Clínico San Carlos, IdISSC CIBERSAM, School of Medicine, Universidad Complutense, Madrid, Spain. ${ }^{8}$ Department of Child and Adolescent Psychiatry, Institute of Psychiatry and Mental Health, Hospital General Universitario Gregorio Marañón, Madrid, Spain. ${ }^{9}$ Department of Primary Education, University of Ioannina, loannina, Greece. ${ }^{10}$ Faculté de Médecine, Université Paris Descartes, Paris, France. ${ }^{11}$ Department of Psychology, Institute of Psychiatry, Psychology and Neuroscience, King's College London, London, UK. ${ }^{12}$ Department of Forensic and Neurodevelopmental Sciences, Institute of Psychiatry, Psychology and Neuroscience, King's College London, London, UK. ${ }^{13}$ School of Medicine, Universidad Complutense, Madrid, Spain. ${ }^{14}$ Instituto de Investigación Sanitaria Gregorio Marañón, Madrid, Spain. ${ }^{15}$ Centro Investigación Biomédica en Red Salud Mental (CIBERSAM), Madrid, Spain.

Received: 15 April 2021 Accepted: 2 February 2022

Published online: 04 March 2022

\section{References}

1. American Psychiatric A. Diagnostic and statistical manual of mental disorders $\left(\mathrm{DSM}-5^{\circledR}\right)$. American Psychiatric Pub; 2013.

2. Howes OD, Rogdaki M, Findon JL, Wichers RH, Charman T, King BH, et al. Autism spectrum disorder: consensus guidelines on assessment, treatment and research from the British Association for Psychopharmacology. J Psychopharmacol. 2018;32(1):3-29. https://doi.org/10.1177/ 0269881117741766

3. Tromans S, Adams C. Brief report: Autism spectrum disorder: a comprehensive survey of randomized controlled trials. J Autism Dev Disord. 2018;48(9):3228-32.

4. Siafis S. Placebo response in pharmacological and dietary supplement trials of autism spectrum disorder (ASD): systematic review and metaregression analysis. Mol Autism. 2020;11:66.

5. Díaz-Caneja CM, State MW, Hagerman RJ, Jacquemont S, Marín O, Bagni C, et al. A white paper on a neurodevelopmental framework for drug discovery in autism and other neurodevelopmental disorders. Eur
Neuropsychopharmacol. 2021. https://doi.org/10.1016/j.euroneuro. 2021.02.020.

6. Fuentes J, Hervás A, Howlin P. ESCAP practice guidance for autism: a summary of evidence-based recommendations for diagnosis and treatment. Eur Child Adolesc Psychiatry. 2021;30(6):961-84. https://doi.org/ 10.1007/s00787-020-01587-4.

7. Berry-Kravis EM, Lindemann L, Jonch AE, Apostol G, Bear MF, Carpenter $R L$, et al. Drug development for neurodevelopmental disorders: lessons learned from fragile X syndrome. Nat Rev Drug Discov. 2018;17(4):28099. https://doi.org/10.1038/nrd.2017.221.

8. Jobski K, Hofer J, Hoffmann F, Bachmann C. Use of psychotropic drugs in patients with autism spectrum disorders: a systematic review. Acta Psychiatr Scand. 2017;135(1):8-28. https://doi.org/10.1111/acps.12644.

9. Carrasco M, Volkmar FR, Bloch MH. Pharmacologic treatment of repetitive behaviors in autism spectrum disorders: evidence of publication bias. Pediatrics. 2012;129(5):e1301-10. https://doi.org/10.1542/peds. 2011-3285.

10. Sturman N, Deckx L, van Driel ML. Methylphenidate for children and adolescents with autism spectrum disorder. Cochrane Database Syst Rev. 2017;11:011144. https://doi.org/10.1002/14651858.CD011144. pub2.

11. Fung LK, Mahajan R, Nozzolillo A, Bernal P, Krasner A, Jo B, et al. Pharmacologic treatment of severe irritability and problem behaviors in autism a systematic review and meta-analysis. Pediatrics. 2016;137(Supplement 2):S124-35.

12. Hirsch LE, Pringsheim T. Aripiprazole for autism spectrum disorders (ASD). Cochrane Database Syst Rev. 2016;2016(6):CD009043. https:// doi.org/10.1002/14651858.CD009043.pub3.

13. Williams K, Brignell A, Randall M, Silove N, Hazell P. Selective serotonin reuptake inhibitors (SSRIs) for autism spectrum disorders (ASD). Cochrane Database Syst Rev. 2013;2013(8):CD004677. https://doi.org/ 10.1002/14651858.CD004677.pub3.

14. Hurwitz R, Blackmore R, Hazell P, Williams K, Woolfenden S. Tricyclic antidepressants for autism spectrum disorders (ASD) in children and adolescents. Cochrane Database Syst Rev. 2012;2012(3):CD008372. https://doi.org/10.1002/14651858.CD008372.pub2.

15. Nye C, Brice A. Combined vitamin B6-magnesium treatment in autism spectrum disorder. Cochrane Database Syst Rev. 2005;2005(4):CD003497. https://doi.org/10.1002/14651858.CD003497. pub2.

16. Fraguas D, Díaz-Caneja CM, Pina-Camacho L, Moreno C, Durán-Cutilla $M$, Ayora $M$, et al. Dietary interventions for autism spectrum disorder: a meta-analysis. Pediatrics. 2019;144:e20183218.

17. Zhou MS, Nasir M, Farhat LC, Kook M, Artukoglu BB, Bloch MH. Metaanalysis: pharmacologic treatment of restricted and repetitive behaviors in autism spectrum disorders. J Am Acad Child Adolesc Psychiatry. 2020. https://doi.org/10.1016/j.jaac.2020.03.007.

18. Fallah MS, Shaikh MR, Neupane B, Rusiecki D, Bennett TA, Beyene J. Atypical antipsychotics for irritability in pediatric autism: a systematic review and network meta-analysis. J Child Adolesc Psychopharmacol. 2019;29(3):168-80. https://doi.org/10.1089/cap.2018.0115.

19. Rodrigues R, Lai MC, Beswick A, Gorman DA, Anagnostou E, Szatmari $P$, et al. Practitioner review: pharmacological treatment of attentiondeficit/hyperactivity disorder symptoms in children and youth with autism spectrum disorder: a systematic review and meta-analysis. J Child Psychol Psychiatry. 2020. https://doi.org/10.1111/jcpp.13305.

20. Hutton B, Catala-Lopez F, Moher D. The PRISMA statement extension for systematic reviews incorporating network meta-analysis: PRISMA-NMA. Med Clin. 2016;147(6):262-6.

21. Masi A, Lampit A, DeMayo MM, Glozier N, Hickie IB, Guastella AJ. A comprehensive systematic review and meta-analysis of pharmacological and dietary supplement interventions in paediatric autism: moderators of treatment response and recommendations for future research. Psychol Med. 2017;47(7):1323-34. https://doi.org/10.1017/S003329171 6003457.

22. Masi A, Lampit A, Glozier N, Hickie IB, Guastella AJ. Predictors of placebo response in pharmacological and dietary supplement treatment trials in pediatric autism spectrum disorder: a meta-analysis. Transl Psychiatry. 2015;5(9): e640. https://doi.org/10.1038/tp.2015.143.

23. Williamson E, Sathe NA, Andrews JC, Krishnaswami S, MCPheeters ML, Fonnesbeck C, et al. AHRQ comparative effectiveness reviews. Medical 
therapies for children with autism spectrum disorder-an update. Agency for Healthcare Research and Quality; 2017.

24. Song L, Luo X, Jiang Q, Chen Z, Zhou L, Wang D, et al. Vitamin D supplementation is beneficial for children with autism spectrum disorder: a meta-analysis. Clin Psychopharmacol Neurosci. 2020;18(2):203-13. https://doi.org/10.9758/cpn.2020.18.2.203.

25. Higgins JPT, Thomas J, Chandler J, Cumpston M, Li T, Page MJ, et al. Cochrane handbook for systematic reviews of interventions. Wiley; 2019.

26. Higgins JPT, Altman DG, Gøtzsche PC, Jüni P, Moher D, Oxman AD, et al. The cochrane collaboration's tool for assessing risk of bias in randomised trials. BMJ. 2011;343:d5928.

27. Devereaux PJ, Choi PT, El-Dika S, Bhandari M, Montori VM, Schünemann $\mathrm{HJ}$, et al. An observational study found that authors of randomized controlled trials frequently use concealment of randomization and blinding, despite the failure to report these methods. J Clin Epidemiol. 2004;57(12):1232-6. https://doi.org/10.1016/j.jclinepi.2004.03.017.

28. Hill CL, LaValley MP, Felson DT. Discrepancy between published report and actual conduct of randomized clinical trials. J Clin Epidemiol. 2002;55(8):783-6. https://doi.org/10.1016/s0895-4356(02)00440-7.

29. Soares HP, Daniels S, Kumar A, Clarke M, Scott C, Swann S, et al. Bad reporting does not mean bad methods for randomised trials: observational study of randomised controlled trials performed by the Radiation Therapy Oncology Group. BMJ. 2004;328(7430):22-4. https://doi.org/10. 1136/bmj.328.7430.22.

30. Mhaskar R, Djulbegovic B, Magazin A, Soares HP, Kumar A. Published methodological quality of randomized controlled trials does not reflect the actual quality assessed in protocols. J Clin Epidemiol. 2012;65(6):602-9. https://doi.org/10.1016/j.jclinepi.2011.10.016.

31. Elbourne DR, Altman DG, Higgins JPT, Curtin F, Worthington HV, Vail A. Meta-analyses involving cross-over trials: methodological issues. Int J Epidemiol. 2002;31(1):140-9.

32. Reichow B, Volkmar FR, Cicchetti DV. Development of the evaluative method for evaluating and determining evidence-based practices in autism. J Autism Dev Disord. 2008;38(7):1311-9.

33. Furukawa TA, Salanti G, Atkinson LZ, Leucht S, Ruhe HG, Turner EH, et al. Comparative efficacy and acceptability of first-generation and secondgeneration antidepressants in the acute treatment of major depression: protocol for a network meta-analysis. BMJ Open. 2016;6(7): e010919. https://doi.org/10.1136/bmjopen-2015-010919.

34. Aman MG, Singh NN, Stewart AW, Field CJ. Psychometric characteristics of the aberrant behavior checklist. Am J Ment Defic. 1985;89(5):492-502.

35. Sparrow SS. Vineland adaptive behavior scales. In: Kreutzer JS, DeLuca J, Caplan B, editors. Encyclopedia of clinical neuropsychology. Springer; 2011. p. 2618-21.

36. Scahill L, McDougle CJ, Williams SK, Dimitropoulos A, Aman MG McCracken JT, et al. Children's Yale-Brown Obsessive Compulsive Scale modified for pervasive developmental disorders. J Am Acad Child Adolesc Psychiatry. 2006;45(9):1114-23. https://doi.org/10.1097/01.chi. 0000220854.79144.e7.

37. Constantino JN, Gruber CP. Social Responsiveness Scale: SRS-2 Software Kit. Western Psychological Services 2012.

38. Schopler E, Reichler RJ, DeVellis RF, Daly K. Toward objective classification of childhood autism: childhood Autism Rating Scale (CARS). J Autism Dev Disord. 1980;10(1):91-103. https://doi.org/10.1007/BF024 08436.

39. McCracken JT, Anagnostou E, Arango C, Dawson G, Farchione T, Mantua $\checkmark$, et al. Drug development for autism spectrum disorder (ASD): progress, challenges, and future directions. Eur Neuropsychopharmacol. 2021;48:3-31. https://doi.org/10.1016/j.euroneuro.2021.05.010.

40. Guy W. CGI. Clinical global impressions. ECDEU assessment manual for psychopharmacology. 1976.

41. Schwarzer G. Meta: an R package for meta-analysis. R News. 2007;7(3):40-5.

42. Rücker G, Schwarzer G, Krahn U, König J, Schwarzer MG. Package'netmeta'. network meta-analysis using frequentist methods (version 07-0). 2015.

43. R Core Team. R: a language and environment for statistical computing R Core Team; 2019.
44. Nikolakopoulou A, Higgins JPT, Papakonstantinou T, Chaimani A, Del Giovane C, Egger M, et al. CINeMA: an approach for assessing confidence in the results of a network meta-analysis. PLoS Med. 2020;17(4): e1003082. https://doi.org/10.1371/journal.pmed.1003082.

45. Papakonstantinou T, Nikolakopoulou A, Higgins JPT, Egger M, Salanti G. CINeMA: software for semiautomated assessment of the confidence in the results of network meta-analysis. Campbell Syst Rev. 2020;16(1): e1080. https://doi.org/10.1002/cl2.1080.

46. Bakbergenuly I, Hoaglin DC, Kulinskaya E. Pitfalls of using the risk ratio in meta-analysis. Res Synth Methods. 2019;10(3):398-419. https://doi. org/10.1002/jrsm.1347.

47. Doi SA, Furuya-Kanamori L, Xu C, Lin L, Chivese T, Thalib L. Questionable utility of the relative risk in clinical research: a call for change to practice. J Clin Epidemiol. 2020. https://doi.org/10.1016/j.jclinepi.2020.08.019.

48. Rücker G, Schwarzer G. Ranking treatments in frequentist network meta-analysis works without resampling methods. BMC Med Res Methodol. 2015;15(1):58. https://doi.org/10.1186/s12874-015-0060-8.

49. Samara MT, Spineli LM, Furukawa TA, Engel RR, Davis JM, Salanti G, et al. Imputation of response rates from means and standard deviations in schizophrenia. Schizophr Res. 2013;151(1-3):209-14. https://doi.org/10. 1016/j.schres.2013.10.029.

50. Siafis S, Rodolico A, Çıray O, Murphy DG, Parellada M, Arango C, et al. Imputing the number of responders from the mean and standard deviation of CGI-improvement in clinical trials investigating medications for autism spectrum disorder. Brain Sci. 2021;11(7):908. https://doi. org/10.3390/brainsci11070908.

51. Balk EM, Earley A, Patel K, Trikalinos TA, Dahabreh IJ. Empirical assessment of within-arm correlation imputation in trials of continuous outcomes. 2012.

52. European Medicines Agency. Guideline on the clinical development of medicinal products for the treatment of Autism Spectrum Disorder (ASD). 2018. p. 1-13.

53. Rhodes KM, Turner RM, Higgins JPT. Predictive distributions were developed for the extent of heterogeneity in meta-analyses of continuous outcome data. J Clin Epidemiol. 2015;68(1):52-60. https://doi.org/10. 1016/j.jclinepi.2014.08.012.

54. Turner RM, Davey J, Clarke MJ, Thompson SG, Higgins JP. Predicting the extent of heterogeneity in meta-analysis, using empirical data from the Cochrane Database of Systematic Reviews. Int J Epidemiol. 2012;41(3):818-27. https://doi.org/10.1093/ije/dys041.

55. Efthimiou O, Debray TPA, van Valkenhoef G, Trelle S, Panayidou K, Moons KGM, et al. GetReal in network meta-analysis: a review of the methodology. Res Synth Methods. 2016;7(3):236-63. https://doi.org/10. 1002/jrsm.1195.

56. Chaimani A, Higgins JPT, Mavridis D, Spyridonos P, Salanti G. Graphical tools for network meta-analysis in STATA. PLoS ONE. 2013;8(10): e76654. https://doi.org/10.1371/journal.pone.0076654.

57. Panagiotou OA, Contopoulos-loannidis DG, loannidis JP. Comparative effect sizes in randomised trials from less developed and more developed countries: meta-epidemiological assessment. BMJ. 2013;346: f707. https://doi.org/10.1136/bmj.f707.

58. Stivaros S, Garg S, Tziraki M, Cai Y, Thomas O, Mellor J, et al. Randomised controlled trial of simvastatin treatment for autism in young children with neurofibromatosis type 1 (SANTA). Mol Autism. 2018;9:12. https:// doi.org/10.1186/s13229-018-0190-z.

59. Nikvarz N, Alaghband-Rad J, Tehrani-Doost M, Alimadadi A, Ghaeli P. Comparing efficacy and side effects of memantine vs. risperidone in the treatment of autistic disorder. Pharmacopsychiatry. 2017;50(1):1925. https://doi.org/10.1055/s-0042-108449.

60. Kent JM, Kushner S, Ning X, Karcher K, Ness S, Aman M, et al. Risperidone dosing in children and adolescents with autistic disorder: a double-blind, placebo-controlled study. J Autism Dev Disord. 2013;43(8):1773-83.

61. NCT01624675. A study to evaluate the efficacy and safety of risperidone (R064766) in children and adolescents with irritability associated with autistic disorder. 2015.

62. McDougle CJ, Scahill L, Aman MG, McCracken JT, Tierney E, Davies M, et al. Risperidone for the core symptom domains of autism: results from the study by the autism network of the research units on pediatric psychopharmacology. Am J Psychiatry. 2005;162(6):1142-8. https://doi. org/10.1176/appi.ajp.162.6.1142. 
63. NCT01171937. Risperidone treatment in children with autism spectrum disorder and high levels of repetitive behavior (ProjectV). 2017.

64. Sokolova E, Oerlemans AM, Rommelse NN, Groot P, Hartman CA, Glennon JC, et al. A causal and mediation analysis of the comorbidity between attention deficit hyperactivity disorder (ADHD) and autism spectrum disorder (ASD). J Autism Dev Disord. 2017;47(6):1595-604. https://doi.org/10.1007/s10803-017-3083-7.

65. Hollander E, Soorya L, Chaplin W, Anagnostou E, Taylor BP, Ferretti CJ, et al. A double-blind placebo-controlled trial of fluoxetine for repetitive behaviors and global severity in adult autism spectrum disorders. Am J Psychiatry. 2012;169(3):292-9. https://doi.org/10.1176/appi.ajp.2011. 10050764.

66. McDougle CJ, Naylor ST, Cohen DJ, Volkmar FR, Heninger GR, Price LH. A double-blind, placebo-controlled study of fluvoxamine in adults with autistic disorder. Arch Gen Psychiatry. 1996;53(11):1001-8. https://doi. org/10.1001/archpsyc.1996.01830110037005.

67. Brandenburg C, Blatt GJ. Differential serotonin transporter (5-HTT) and 5-HT(2) receptor density in limbic and neocortical areas of adults and children with autism spectrum disorders: implications for selective serotonin reuptake inhibitor efficacy. J Neurochem. 2019;151(5):642-55. https://doi.org/10.1111/jnc.14832.

68. Pandina G, Ring RH, Bangerter A, Ness S. Current approaches to the pharmacologic treatment of core symptoms across the lifespan of autism spectrum disorder. Psychiatr Clin. 2020;43(4):629-45. https://doi. org/10.1016/j.psc.2020.08.003.

69. Hollander E, Soorya L, Wasserman S, Esposito K, Chaplin W, Anagnostou E. Divalproex sodium vs. placebo in the treatment of repetitive behaviours in autism spectrum disorder. Int J Neuropsychopharmacol. 2006:9(2):209-13.

70. Hollander E, Chaplin W, Soorya L, Wasserman S, Novotny S, Rusoff J, Feirsen N, Pepa L, Anagnostou E. Divalproex sodium vs placebo for the treatment of irritability in children and adolescents with autism spectrum disorders. Neuropsychopharmacology. 2010;35(4):990-8.

71. Hellings JA, Weckbaugh M, Nickel EJ, Cain SE, Zarcone JR, Reese RM, Hall S, Ermer DJ, Tsai LY, Schroeder SR, Cook EH. A Double-Blind, Placebo-Controlled Study of Valproate for Aggression in Youth with Pervasive Developmental Disorders. J Child Adolesc Psychopharmacol. 2005;15(4):682-92.

72. Wasserman S, Iyengar R, Chaplin WF, Watner D, Waldoks SE, Anagnostou E, et al. Levetiracetam versus placebo in childhood and adolescent autism: a double-blind placebo-controlled study. Int Clin Psychopharmacol. 2006;21(6):363-7. https://doi.org/10.1097/01.yic.0000224787. 13782.0f.

73. Chen B, Choi H, Hirsch LJ, Katz A, Legge A, Buchsbaum R, et al. Psychiatric and behavioral side effects of antiepileptic drugs in adults with epilepsy. Epilepsy Behav. 2017;76:24-31. https://doi.org/10.1016/j. yebeh.2017.08.039.

74. McLay LL, France KG, Blampied NM, Hunter JE, van Deurs JR, Woodford EC, et al. Collateral child and parent effects of function-based behavioral interventions for sleep problems in children and adolescents with autism. J Autism Dev Disord. 2021. https://doi.org/10.1007/ s10803-021-05116-3.

75. Hunter JE, McLay LK, France KG, Blampied NM. Systematic review of the collateral effects of behavioral sleep interventions in children and adolescents with autism spectrum disorder. Res Autism Spectrum Disord. 2020;79: 101677. https://doi.org/10.1016/j.rasd.2020.101677.

76. Lacivita E, Perrone R, Margari L, Leopoldo M. Targets for drug therapy for autism spectrum disorder: challenges and future directions. J Med Chem. 2017;60(22):9114-41. https://doi.org/10.1021/acs.jmedchem. 7 b00965.

77. Díaz-Caneja CM, State MW, Hagerman RJ, Jacquemont S, Marín O, Bagni C, et al. A white paper on a neurodevelopmental framework for drug discovery in autism and other neurodevelopmental disorders. Eur Neuropsychopharmacol. 2021;48:49-88. https://doi.org/10.1016/j. euroneuro.2021.02.020.

78. Baribeau D, Anagnostou E. Novel treatments for autism spectrum disorder based on genomics and systems biology. Pharmacol Ther. 2021;230:107939. https://doi.org/10.1016/j.pharmthera.2021.107939.

79. Persico AM, Ricciardello A, Lamberti M, Turriziani L, Cucinotta F, Brogna $C$, et al. The pediatric psychopharmacology of autism spectrum disorder: a systematic review_part I: the past and the present. Prog
Neuropsychopharmacol Biol Psychiatry. 2021;110: 110326. https://doi. org/10.1016/j.pnpbp.2021.110326.

80. NCT02901431. A Study to investigate the efficacy and safety of Balovaptan (ro5285119) in Participants With Autism Spectrum Disorder (ASD). 2020.

81. Sikich L, Kolevzon A, King BH, McDougle CJ, Sanders KB, Kim SJ, et al. Intranasal oxytocin in children and adolescents with autism spectrum disorder. N Engl J Med. 2021;385(16):1462-73. https://doi.org/10.1056/ NEJMoa2103583.

82. Freeman $\mathrm{SM}$, Palumbo MC, Lawrence $\mathrm{RH}$, Smith $\mathrm{AL}$, Goodman MM, Bales KL. Effect of age and autism spectrum disorder on oxytocin receptor density in the human basal forebrain and midbrain. Transl Psychiatry. 2018;8(1):257. https://doi.org/10.1038/s41398-018-0315-3.

83. John S, Jaeggi AV. Oxytocin levels tend to be lower in autistic children: a meta-analysis of 31 studies. Autism. 2021;25(8):2152-61. https://doi. org/10.1177/13623613211034375.

84. Bolognani F, Del Valle RM, Squassante L, Wandel C, Derks M, Murtagh $L$, et al. A phase 2 clinical trial of a vasopressin $V 1$ a receptor antagonist shows improved adaptive behaviors in men with autism spectrum disorder. Sci Transl Med. 2019;11(491):eaat7838. https://doi.org/10.1126/ scitranslmed.aat7838.

85. NCT03504917. A study of balovaptan in adults with autism spectrum disorder with a 2-year open-label extension. 2020.

86. Parker KJ, Oztan O, Libove RA, Mohsin N, Karhson DS, Sumiyoshi RD, et al. A randomized placebo-controlled pilot trial shows that intranasal vasopressin improves social deficits in children with autism. Sci Transl Med. 2019;11(491):eaau356. https://doi.org/10.1126/scitranslmed.aau73 56.

87. Crutel V, Lambert E, Penelaud PF, Albarrán Severo C, Fuentes J, Rosier A, et al. Bumetanide oral liquid formulation for the treatment of children and adolescents with autism spectrum disorder: design of two phase III studies (SIGN trials). J Autism Dev Disord. 2020. https://doi.org/10.1007/ s10803-020-04709-8.

88. Neurochlore S. Servier and Neurochlore announce the main results of the two phase 3 clinical studies assessing bumetanide in the treatment of autism spectrum disorders in children and adolescents. https:// servier.com/en/communique/servier-and-neurochlore-announcethe-main-results-of-the-two-phase-3-clinical-studies-assessing-bumet anide-in-the-treatment-of-autism-spectrum-disorders-in-children-andadolescents/. https://servier.com/en/communique/servier-and-neuro chlore-announce-the-main-results-of-the-two-phase-3-clinical-studi es-assessing-bumetanide-in-the-treatment-of-autism-spectrum-disor ders-in-children-and-adolescents/ (2021). Accessed 07.09.2021.

89. Aran A, Harel M, Cassuto H, Polyansky L, Schnapp A, Wattad N, et al. Cannabinoid treatment for autism: a proof-of-concept randomized trial. Mol Autism. 2021;12(1):6. https://doi.org/10.1186/s13229-021-00420-2.

90. Campbell M, Anderson LT, Small AM, Adams P, Gonzalez NM, Ernst M. Naltrexone in autistic children: behavioral symptoms and attentional learning. J Am Acad Child Adolesc Psychiatry. 1993;32(6):1283-91. https://doi.org/10.1097/00004583-199311000-00024.

91. Anagnostou E. NCT03887676 Arbaclofen vs. placebo in the treatment of children and adolescents with ASD (ARBA) (2019).

92. Parellada M, San José Cáceres A, Palmer M, Delorme R, Jones EJH, Parr $J R$, et al. A phase II randomized, double-blind, placebo-controlled study of the efficacy, safety, and tolerability of arbaclofen administered for the treatment of social function in children and adolescents with autism spectrum disorders: study protocol for AIMS-2-TRIALS-CT1. Front Psychiatry. 2021;12:701729. https://doi.org/10.3389/fpsyt.2021.701729.

93. Gagan J. NCT03553875. Memantine for the treatment of social deficits in youth with disorders of impaired social interactions. 2018.

94. GW Research Ltd. NCT04745026. Trial to investigate the safety and efficacy of cannabidiol oral solution (GWP42003-P; CBD-OS) in children and adolescents with autism spectrum disorder. 2021.

95. Hollander E. NCT03202303. Cannabidivarin (CBDV) vs. placebo in children with autism spectrum disorder (ASD). 2017.

96. Anagnostou E, Bennett TA, Thorpe K, Nicolson R. 5.16 A phase 2 randomized, placebo-controlled trial of Tideglusib, an orally administered GSK-3 beta inhibitor, in the treatment of adolescents with ASD. J Am Acad Child Adolesc Psychiatry. 2018;57(10):S232. https://doi.org/10. 1016/j.jaac.2018.09.311. 
97. Anagnostou E. NCT01661855. A pilot study of riluzole versus placebo in the treatment of children and adolescents with ASD (RILISE). 2017.

98. Rothman J. L1-79 and the role of catecholamines in autism. Autism spectrum disorder-profile, heterogeneity, neurobiology and intervention. 2020.

99. Frye R. NCT02839915. Leucovorin for the treatment of language impairment in children with autism spectrum disorder. 2016.

100. Novotny S. NCT01260961. Developing treatment, treatment validation and treatment scope in the setting of an autism clinical trial. 2010.

101. Zimmerman AW, Singh K, Connors SL, Liu H, Panjwani AA, Lee LC, et al. Randomized controlled trial of sulforaphane and metabolite discovery in children with autism spectrum disorder. Mol Autism. 2021;12(1):38. https://doi.org/10.1186/s13229-021-00447-5.

102. Singh K, Connors SL, Macklin EA, Smith KD, Fahey JW, Talalay P, et al. Sulforaphane treatment of autism spectrum disorder (ASD). Proc Natl Acad Sci. 2014;111(43):15550. https://doi.org/10.1073/pnas.1416940111.

103. NCT02909959. Sulforaphane for the treatment of young men with autism spectrum disorder. 2020.

104. Smith R, Ou J, Jin H, Wu R, Fahey JW, Arriaza J, et al. M22. Sulforaphane as a treatment for autism: a randomized double-blind study. ACNP 59th annual meeting: poster session I. Neuropsychopharmacology. 2020;45(1):79-80. https://doi.org/10.1038/s41386-020-00890-7

105. Castejon AM, Spaw JA, Rozenfeld I, Sheinberg N, Kabot S, Shaw A, et al. Improving antioxidant capacity in children with autism: a randomized, double-blind controlled study with cysteine-rich whey protein. Front Psychiatry. 2021;12: 669089. https://doi.org/10.3389/fpsyt.2021.669089.

106. Hendren RL, James SJ, Widjaja F, Lawton B, Rosenblatt A, Bent S. Randomized, placebo-controlled trial of methyl B12 for children with autism. J Child Adolesc Psychopharmacol. 2016;26(9):774-83. https://doi.org/ 10.1089/cap.2015.0159.

107. Rossignol DA, Frye RE. The effectiveness of cobalamin (B12) treatment for autism spectrum disorder: a systematic review and meta-analysis. $J$ Pers Med. 2021;11(8):784. https://doi.org/10.3390/jpm11080784.

108. Debray TP, Moons KG, van Valkenhoef G, Efthimiou O, Hummel N, Groenwold RH, et al. Get real in individual participant data (IPD) meta-analysis: a review of the methodology. Res Synth Methods. 2015;6(4):293-309. https://doi.org/10.1002/jrsm.1160.

109. Chugani DC, Chugani HT, Wiznitzer M, Parikh S, Evans PA, Hansen RL, et al. Efficacy of low-dose buspirone for restricted and repetitive behavior in young children with autism spectrum disorder: a randomized trial. J Pediatr. 2016;170:45-53.e1-4. https://doi.org/10.1016/j.jpeds.2015. 11.033.

110. Autistica. Top ten questions for autism research. 2016

111. Anixt JS, Murray DS, Coury DL, Kuhlthau KA, Eskra D, Seide J, et al. Improving behavior challenges and quality of life in the autism learning health network. Pediatrics. 2020;145(Suppl 1):S20-9. https://doi.org/10. 1542/peds.2019-1895E.

112. Solmi M, Fornaro M, Ostinelli EG, Zangani C, Croatto G, Monaco F, et al. Safety of 80 antidepressants, antipsychotics, anti-attention-deficit/ hyperactivity medications and mood stabilizers in children and adolescents with psychiatric disorders: a large scale systematic meta-review of 78 adverse effects. World Psychiatry. 2020;19(2):214-32. https://doi.org/ 10.1002/wps.20765.

113. Lemonnier E, Villeneuve N, Sonie S, Serret S, Rosier A, Roue M, et al. Effects of bumetanide on neurobehavioral function in children and adolescents with autism spectrum disorders. Transl Psychiatry. 2017;7(5): e1124. https://doi.org/10.1038/tp.2017.101.

114. Kloosterboer SM, de Winter BCM, Reichart CG, Kouijzer MEJ, de Kroon MMJ, van Daalen E, et al. Risperidone plasma concentrations are associated with side effects and effectiveness in children and adolescents with autism spectrum disorder. Br J Clin Pharmacol. 2020. https://doi. org/10.1111/bcp.14465.

\section{Publisher's Note}

Springer Nature remains neutral with regard to jurisdictional claims in published maps and institutional affiliations. 